\title{
Síntese de compósito a base de quitosana e sílica para adsorção de corante têxtil
}

As técnicas usuais de tratamento de efluentes demandam de tecnologias complementares para remover de forma eficiente os contaminantes mais persistente do meio, como é o caso dos corantes. Para a remoção desses compostos a adsorção tem se demonstrado efetiva, entretanto, requer materiais adsorventes com propriedades físicas e químicas favoráveis. Com isso, o desenvolvimento de compósitos a base de quitosana e sílica têm sido investigados para melhorar as características dos adsorventes e aumentar a capacidade de adsorção, entretanto, ainda é um desafio. Desta forma, este estudo teve como objetivo desenvolver um compósito adsorvente à base de quitosana pelo processo sol-gel utilizando como precursor tetraetoxissilano e verificando a capacidade deste compósito em remover corante têxtil em solução aquosa. O compósito foi desenvolvido a partir de uma relação de D-glucosamina:TEOS de 2,77:1. O material foi caracterizado quimicamente, verificando a preponderância de grupamentos funcionais da quitosana e ponto de carga zero em $\mathrm{pH} 8,90$. O desempenho do compósito foi avaliado através de isotermas e cinéticas de adsorção do corante têxtil Azul Brilhante de Remazol R. Os dados de equilíbrio foram melhor correlacionados através do modelo isotérmico de Liu $(R 2>0,97)$. As cinéticas de adsorção foram preditas pelo modelo de Elovich (R2>0,97). O desenvolvimento do compósito foi efetivo para remover o corante com elevada capacidade de adsorção e preferencialmente por atrações eletrostáticas.

Palavras-chave: Adsorvente; Sol-gel; TEOS; Xerogel; Azul Brilhante de Remazol R.

\section{Synthesis of composite based on chitosan and silica for adsorption of textile dye}

\begin{abstract}
The usual techniques for treating effluents require complementary technologies to remove efficiently the most persistent contaminants from the environment such as dyes. For the removal of these compounds, adsorption has been shown to be effective, however, it requires adsorbent materials with favorable physical and chemical properties. Thus, the development of chitosan and silica-based composites has been investigated to improve the characteristics of the adsorbents and increase the adsorption capacity, however, it is still a challenge. Thus, this study aimed to develop a chitosan-based adsorbent composite by the sol-gel process using tetraethoxysilane as a precursor and verifying the capacity of this composite to remove textile dye in aqueous solution. The composite was developed from D-glucosamine:TEOS of 2.77:1 ratio. The material was chemically characterized, checking the preponderance of chitosan functional groups and zero load point in $\mathrm{pH}$ 8.90. The performance of the composite was evaluated using isotherms and adsorption kinetics of the Remazol R bright blue textile dye. The balance data were better correlated through the Liu isothermal model (R2>0.97). The adsorption kinetics were predicted by the Elovich model (R2>0.97). The development of the composite was effective to remove the dye with high adsorption capacity and preferably per electrostatic attractions.
\end{abstract}

Keywords: Adsorbent; Sol-gel; TEOS; Xerogel; Remazol Brilliant Blue R.

Topic: Engenharia Química

Reviewed anonymously in the process of blind peer
Received: 04/03/2021

Approved: 22/03/2021
Thaís Strieder Machado (10) Universidade de Passo Fundo, Brasil http://lattes.cnpq.br/9644112526589073 http://orcid.org/0000-0002-5555-8517 thaiis.strieder@hotmail.com

\section{Flávia Melara (iD}

Universidade de Passo Fundo, Brasil http://lattes.cnpq.br/8212031330105973 http://orcid.org/0000-0002-3785-3491 175626@upf.br

\section{Larissa Crestani (iD)}

Universidade de Passo Fundo, Brasil http://lattes.cnpq.br/7665563197998906 http://orcid.org/0000-0003-2014-9614 http://orcid.org/0000-0003-2

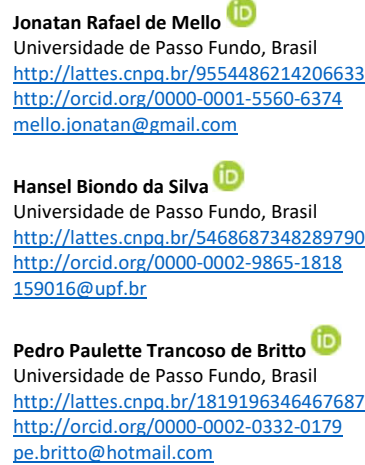

pe.britto@hotmail.com

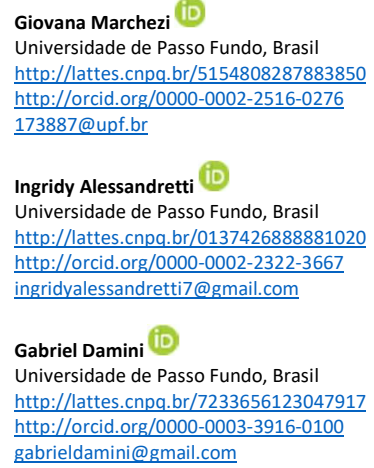

\section{Referencing this:}

MACHADO, T. S.; MELARA, F.; CRESTANI, L.; MELLO, J. R.; SILVA, H. B.; BRITTO, P. P. T.; MARCHEZI, G.; ALESSANDRETTI, I.; DAMINI, G.; PICCIN, J. S.. Síntese de compósito a base de quitosana e sílica para adsorção de corante têxtil. Revista Ibero Americana de Ciências Ambientais, v.12, n.3, p.408-420, 2021. DOI: http://doi.org/10.6008/CBPC2179-6858.2021.003.0033

DOI: 10.6008/CBPC2179-6858.2021.003.0033 


\section{INTRODUÇÃO}

A adsorção apresenta elevado potencial para a remoção de diversos contaminantes persistentes (ELTAWEIL et al., 2020; LIU et al., 2019; GISI et al., 2016), os quais as técnicas convencionais de tratamento de efluentes não são capazes de remover ou degradar (BURAKOV et al., 2018). Nesse sentido, pesquisas têm sido desenvolvidas com o intuito de obter adsorventes com altas capacidades de adsorção a partir de materiais de baixo custo, como resíduos de curtumes (GOMES et al., 2016; PICCIN et al., 2013), cascas de cupuaçu (CARDOSO et al., 2011), resíduos da indústria cervejeira (ROSSI et al., 2018), quitosana obtida de resíduos da indústria pesqueira (FRANCO et al., 2015), entre outros. Entretanto, a busca por materiais com propriedades físicas e químicas favoráveis à adsorção é um desafio, como por exemplo, adsorventes com capacidade de remoção em várias condições experimentais, alta área superficial e numerosos sítios ativos.

A quitosana ( $\beta$-(1-4)-D-glucosamina) é um biopolímero obtido pela desacetilação alcalina da quitina, através do exoesqueleto de crustáceos ou da parede celular de fungos (MOURA et al., 2015; VAKILI et al., 2014). A reação de desacetilação consiste na remoção de grupos acetil (N-acetil-D-glucosamina) pela substituição por grupos amina $\left(\mathrm{NH}_{2}\right)$ (MOURA et al., 2015), sendo determinada por grau de desacetilação e, assim, permitindo a distinção entre quitina e quitosana. A variação deste grau gera diferenças nas suas propriedades, como solubilidade, biodegradação e principalmente na presença de grupos amina ionizáveis, o que altera a capacidade de interação com outras moléculas químicas (LIZARDI-MENDOZA et al., 2016).

$\mathrm{Na}$ área ambiental, a quitosana pode ser aplicada como adsorvente para o polimento final de efluentes contendo corantes (PICCIN et al., 2009), metais (KOLODYNSKA, 2011) e fármacos (LIU et al., 2019; LESSA et al., 2018; KYZAS et al., 2013). Em contrapartida, a quitosana na sua forma natural apresenta porosidade e área superficial incompatíveis com adsorventes comerciais, além de ser solúvel em meios ácidos, o que dificulta o seu uso in natura em sistemas de adsorção (FUTALAN et al., 2011). Com isso, modificações na quitosana podem ser efetivas na superação dessas características indesejáveis do material.

Um tipo de modificação proposto é a formação de compósitos (NGAH et al., 2011), através da interação com outros materiais para melhorar as propriedades físicas e químicas da quitosana. Com isso, precursores de sílica através do processo sol-gel têm sido aplicados para o desenvolvimento de compósitos adsorventes (BUDNYAK et al., 2015). A adição da sílica em conjunto à quitosana, pode oferecer estabilidade em meios ácidos e em diferentes temperaturas (BUDNYAK et al., 2016), originando um compósito com características desejáveis.

O processo sol-gel é um método em que uma solução sol (solução coloidal com partículas variando entre 1 e $100 \mathrm{~nm}$ ), passa para um gel gerando uma rede rígida e porosa de dimensões submicrométricas (BUCKLEY et al., 1994). O gel de sílica é formado pelas reações simultâneas de hidrólise dos grupos etoxissilanos $\left(\mathrm{Si}_{(}\left(\mathrm{OC}_{2} \mathrm{H}_{5}\right)\right)$ e policondensação de etoxissilanos e silanol $(\mathrm{Si}-\mathrm{OH})$ do precursor de sílica (ZOU et al., 2008; BUCKLEY et al., 1994; HENCH et al., 1990; KLEIN, 1985) sendo o tetraetoxissilano (TEOS) o mais utilizado. Então, são formados grupos $\equiv \mathrm{Si}-\mathrm{O}-\mathrm{Si} \equiv$, conferindo uma estrutura tridimensional e uma rede porosa interconectada (COPELLO et al., 2014; ZOU et al., 2008; BUCKLEY et al., 1994). 
Assim, o objetivo desse estudo foi desenvolver um compósito adsorvente a base de quitosana pelo processo sol-gel utilizando como precursor tetraetoxissilano e verificando a capacidade deste compósito em remover corante têxtil em solução aquosa. Para isso, o compósito adsorvente foi analisado quanto aos seus grupos funcionais, ponto de carga zero, e realizadas isotermas e cinéticas de adsorção em solução aquosa a fim de determinar a sua capacidade de adsorção frente ao corante Azul Brilhante de Remazol R.

\section{MATERIAIS E MÉTODOS}

\section{Obtenção da quitosana}

A quitosana foi obtida a partir de cascas de camarão pelo procedimento descrito por Weska et al. (2007), com as adaptações sugeridas por Moura et al. (2015). As cascas de camarão foram desmineralizadas mantendo-as em agitação por 2 horas em ácido clorídrico $(\mathrm{HCl})$ 2,5\% (v/v) e lavadas. Após foram desproteinizadas em solução de hidróxido de sódio $(\mathrm{NaOH}) 5 \%(\mathrm{~m} / \mathrm{v})$ em agitação por 2 horas. A desodorização foi realizada com solução de hipoclorito de sódio ( $\mathrm{NaClO}$ ) 0,36\% (v/v), em agitação por 3 horas, dando origem a quitina que foi, então, lavada com água em abundância, seca por convecção $\left(60^{\circ} \mathrm{C}\right.$ por $24 \mathrm{~h}$, Tecnal, TE-394) e triturada.

A desacetilação termoquímica da quitina foi realizada em um reator com refluxo aberto (confecção própria, de inox, com diâmetro de $22 \mathrm{~cm}$ e capacidade útil de 6 L) acoplado com agitador mecânico, com solução de $\mathrm{NaOH} 45 \%$ (m/v). O sistema foi aquecido na temperatura de ebulição da solução, permanecendo por 4 horas. Após o material foi lavado até pH próximo ao neutro $(7,5$ a 8,0), obtendo-se a quitosana.

A quitosana foi purificada através da solubilização em solução de ácido acético $2 \%$ (v/v), em agitação por aproximadamente 12 horas. Esta solução foi centrifugada a 3500 rpm por 20 min (Eppendorf, modelo 5810r) e o material sólido foi descartado. O sobrenadante teve seu pH ajustado para $12 \mathrm{com} \mathrm{NaOH} 2 \mathrm{~mol} / \mathrm{L}$. Nesta condição, a quitosana obtida foi precipitada do sobrenadante e a suspensão teve seu pH ajustado para 7,5 com HCl $1 \mathrm{~mol} / \mathrm{L}$ e foi centrifugada a $3500 \mathrm{rpm}$ por $20 \mathrm{~min}$ (Eppendorf, modelo 5810r), obtendo-se a quitosana purificada úmida. A quitosana foi liofilizada (Terroni, modelo LS 3000) e moída em almofariz. As amostras foram mantidas à temperatura ambiente até seu uso. A quitosana apresentou grau de desacetilação superior a $85 \%$.

\section{Desenvolvimento do compósito adsorvente}

O método de produção do adsorvente seguiu o proposto por Essel et al. (2018), com adaptações. Inicialmente, $3 \mathrm{~g}$ de quitosana foram dissolvidas em $50 \mathrm{~mL}$ de solução de ácido acético $2 \%$. Após, nessa mesma solução, foi adicionado 45,5 mL de água destilada, $3 \mathrm{~mL}$ de solução de $\mathrm{HCL} 0,5 \mathrm{~mol} / \mathrm{L}$ e 1,5 mL de tetraetoxisilano (TEOS), resultando em uma proporção molar de D-glucosamina:TEOS de 2,77:1, e essa mistura foi mantida sob agitação por 2 horas (Fisatom, modelo 713D). Com isso, o gel formado foi gotejado em $250 \mathrm{~mL}$ de solução de $\mathrm{NaOH} 2 \mathrm{~mol} / \mathrm{L}$ sob agitação (Fisatom, modelo 754D) para evitar o colapso das esferas. Posteriormente, o material precipitado foi mantido em agitação por 100 rpm por 12 horas em mesa 
agitadora orbital tipo shaker (Tecnal, modelo TE-421). Após, o material foi lavado com água destilada até pH neutro e seco por convecção paralela de ar a $1,5 \mathrm{~m} / \mathrm{s}$ (Tecnal, modelo TE-394) à $30^{\circ} \mathrm{C}$ por 24 horas.

\section{Caracterização do compósito}

Foi realizada a identificação dos grupos funcionais da quitosana e do compósito adsorvente através da análise de infravermelho com transformada de Fourier - FTIR (Agilent Technologies, modelo Cary 630), utilizando a técnica de Refletância Total Atenuada - FTIR/ATR, a partir de $1 \mathrm{~g}$ do material. Posteriormente, foram analisadas por espectroscopia no infravermelho, na faixa de 4000 a $650 \mathrm{~cm}^{-1}$, escaneado 48 vezes cada espectro com uma resolução de $4 \mathrm{~cm}^{-1}$, e o processamento dos espectros foi realizado com o software MicroLab versão B.5 (Agilent Technologies).

$\mathrm{O}$ ponto de carga zero $\left(\mathrm{pH}_{\mathrm{pcz}}\right)$ foi determinado utilizando o método de desvio de $\mathrm{pH}$, adaptado de Newcombe et al. (1993). Para isso, 0,025 g do adsorvente foram misturados a $25 \mathrm{~mL}$ de solução de $\mathrm{NaCl} 0,05$ $\mathrm{mol} / \mathrm{L}$, na faixa de $\mathrm{pH} 3,0$ a 10,0, ajustados com $\mathrm{NaOH}$ 0,1 mol/L e HCl 0,1 mol/L. Logo, foram mantidos em agitação à $100 \mathrm{rpm}$ (Tecnal, modelo TE-421) por $24 \mathrm{~h}$ e $25^{\circ} \mathrm{C}$, posteriormente foi realizada a leitura do $\mathrm{pH}$ final. $\mathrm{O} \mathrm{pH}_{\mathrm{pcz}}$ corresponde ao $\mathrm{pH}$ que cruzar a linha $\mathrm{pH}$ inicial $=\mathrm{pH}$ final.

\section{Ensaios de adsorção}

Os ensaios de adsorção do corante têxtil Azul Brilhante de Remazol R (ABRR, CAS no 2580-78-1, adquirido da Sigma-Aldrich) foram conduzidos em mesa agitadora termostatizada tipo shaker (Tecnal, modelo TE-421), à $25^{\circ} \mathrm{C}$ e agitação de $100 \mathrm{rpm}$. Os ensaios foram realizados em pH $3(3,0 \pm 0,2)$ utilizando solução tampão Mcllvaine (ácido cítrico $0,1 \mathrm{~mol} / \mathrm{L}$ e fosfato dissódico $0,2 \mathrm{~mol} / \mathrm{L}$ ) para favorecer a protonoção da quitosana. Em todos os ensaios de adsorção as concentrações do corante ABRR nas soluções foram determinadas por espectrofotometria, conforme curva padrão determinada em 595 nm ([C, em $\mathrm{mg} / \mathrm{L}]=113,74 *$ absorbância-1,225, $\mathrm{R}^{2}$ igual a 0,9997).

Para as isotermas de adsorção, 0,05 g de adsorvente e $5 \mathrm{~mL}$ de solução tampão Mcllvaine no pH de estudo foram misturados com tempo de contato de $15 \mathrm{~min}$, e após foi adicionado $50 \mathrm{~mL}$ de solução com corante à temperatura de $25^{\circ} \mathrm{C}$. As concentrações iniciais do corante nos pontos de equilíbrio foram avaliadas na faixa de $50 \mathrm{mg} / \mathrm{L}$ a $400 \mathrm{mg} / \mathrm{L}$. Alíquotas foram coletadas a cada 2 horas até que o equilíbrio na concentração do corante em solução fosse observado. $O$ ensaio cinético de adsorção do corante foi realizado com $0,25 \mathrm{~g}$ do adsorvente misturados a $25 \mathrm{~mL}$ de solução tampão Mcllvaine no $\mathrm{pH}$ de estudo e após 15 minutos foram adicionados $250 \mathrm{~mL}$ da solução com corante contendo $100 \mathrm{mg} / \mathrm{L}$. Foram executadas leituras das concentrações nos tempos de 0 a $360 \mathrm{~min}$.

Os ajustes dos parâmetros dos modelos de isotermas e cinéticas de adsorção aos dados experimentais foram realizados utilizando o Software MATLAB ${ }^{\circledR 1}$.

\footnotetext{
${ }^{1}$ Os algoritmos utilizados na modelagem estão disponíveis em https://www.dropbox.com/sh/elq2br3319ccbds/AAB2CTE2Hgb8bG_0Ue48tfLfa?dl=0 


\section{RESULTADOS E DISCUSSÃO}

\section{Síntese e caracterização do compósito adsorvente}

Os grupos funcionais do adsorvente desenvolvido e da quitosana foram avaliados a partir do espectro de infravermelho, apresentados na Figura 1. A ampla faixa de absorção entre $3500-3200 \mathrm{~cm}^{-1}$ representa os grupos hidroxila das cadeias de D-glicopiranose e as aminas primárias livres (MUÑOZ et al., 2015), sendo atribuídas a sobreposição das vibrações de alongamento O-H e N-H (GOMAA et al., 2018; ABDEL-GAWAD et al., 2017). Os picos em torno de $1500 \mathrm{~cm}^{-1}$ correspondem as aminas secundárias $\left(\mathrm{NH}_{2}\right)$ e os picos próximos a $1400 \mathrm{~cm}^{-1}$ referem-se às bandas C-N. As bandas de absorção próximos a $1000 \mathrm{~cm}^{-1}$ correspondem aos grupamentos C-O de álcool primário. Conforme Repo et al. (2011), picos em torno de 1090-1060 cm ${ }^{-1}$ representam as vibrações Si-O-Si e Si-O-C da formação da rede estruturada do material. As diferenças nas intensidades dos picos dos compostos, indicam a modificação físico-química da quitosana para a formação do material com a rede de sílica (ESSEL et al., 2018; COPELLO et al., 2014).

O adsorvente desenvolvido apresentou coloração característica de quitosana e um aspecto semiesférico, denominado xerogel devido ao solvente ter sido removido por evaporação (PERDIGOTO et al., 2012; KLEIN, 1985). A quitosana interage com os grupamentos de sílica através de ligações covalentes e iônicas, formando uma rede quitosana-sílica (REPO et al., 2011). Os tipos das ligações envolvidas na rede quitosana-sílica são importantes, tendo em vista que os grupos aminas estando disponíveis permitirá a realização de diferentes transformações químicas, pois é um dos principais grupamentos químicos para aplicações em fenômenos de adsorção. Os grupamentos aminas ficam disponíveis através das ligações covalentes. $\mathrm{O} \mathrm{pH}_{\mathrm{PCZ}}$ foi determinado com o intuito de verificar as cargas superficiais do xerogel desenvolvido, sendo apresentado na Figura 2.

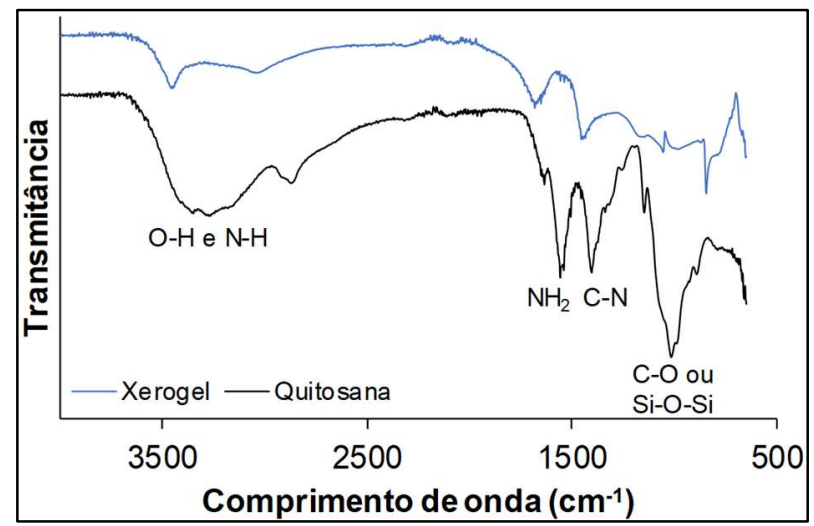

Figura 1: Espectros de infravermelho para o xerogel desenvolvido e a quitosana de origem crustácea.

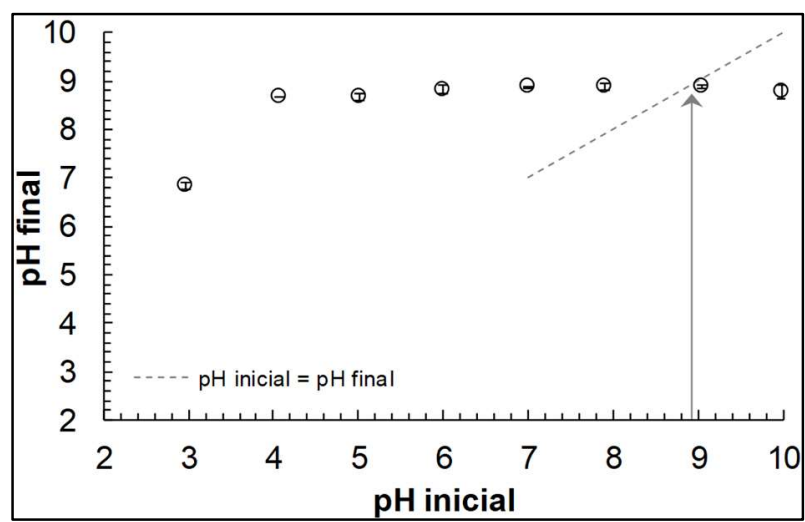

Figura 2: Determinação das cargas superficiais através do ponto de carga zero ( $\mathrm{pH}$ PCZ) para o xerogel desenvolvido.

Conforme a Figura 2, o pH pczé de 8,90 indicando que em pH mais básico que o $\mathrm{pH}_{\mathrm{Pcz}}$ a superfície do material assume cargas negativas e em pH mais ácido que o $\mathrm{pH}$ pcz a superfície do material assume cargas positivas (FIOL et al., 2009). Os grupos aniônicos presentes nas moléculas do corante ABRR, interagem fortemente com os grupos amina originados da protonação da quitosana em meio ácido $\left(\mathrm{NH}_{3}{ }^{+}\right)$, favorecendo 
o fenômeno de adsorção (IBANESCU et al., 2018; DOTTO et al., 2012). A medida que o pH do meio aumenta, menos grupos amina são protonados, mas ainda pode ocorrer adsorção através de interações de Van der Waals e ligações de hidrogênio (BLACKBURN, 2004). Isso já é bem relatado na literatura, como observado por Piccin et al. (2009), Dotto et al. (2012) e Subramani et al. (2017) que utilizaram quitosana in natura para adsorção de corantes e obtiveram melhores resultados em meios ácidos. Dessa forma, em pH ácido a adsorção de ânions é favorecida, indo ao encontro dos ensaios de adsorção terem sido executados em pH 3.

Com isso, na Figura 3 são apresentadas as principais interações responsáveis pela adsorção do corante em meio ácido, onde os grupos protonados da quitosana $\left(\mathrm{NH}_{3}{ }^{+}\right)$possibilitam a interação via atração eletrostática com o grupo trióxido de enxofre $\left(\mathrm{SO}_{3}{ }^{-}\right)$e via ressonância com os anéis aromáticos do corante. Já os grupos hidroxila $\left(\mathrm{OH}^{-}\right)$da quitosana possibilitam a interação via pontes de hidrogênio com os oxigênios (O) e os nitrogênios $(N)$ presentes na estrutura do corante, e ainda via ressonância com os anéis aromáticos do corante.

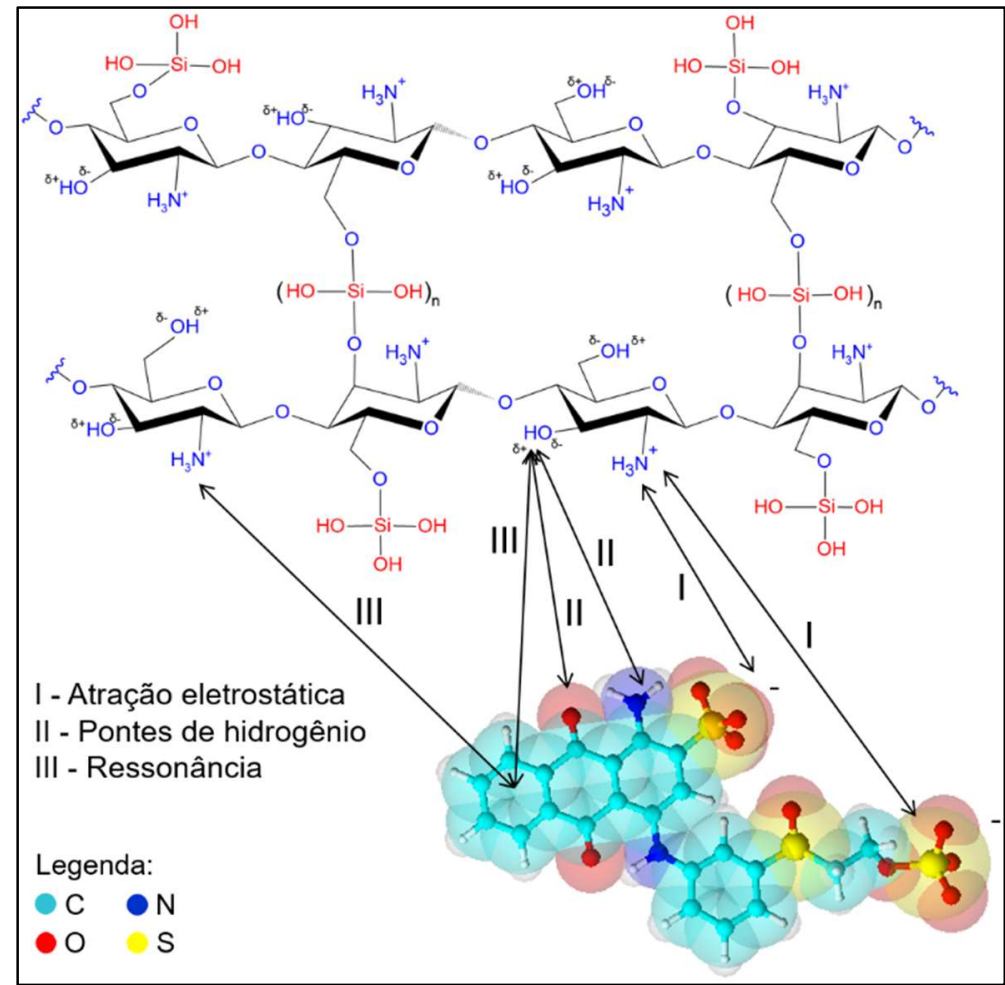

Figura 3: Estruturas químicas sugeridas para os principais mecanismos de adsorção do corante ABRR com o xerogel desenvolvido através da protonação da quitosana.

\section{Ensaios de adsorção}

Para o processo de adsorção do corante ABRR pelo xerogel desenvolvido, é apresentado na Figura 4 a curva de equilíbrio, em conjunto com a representação dos dados através dos modelos de Langmuir (1918), Freundlich (1906) e Liu et al. (2003), pelas Equações 3, 4 e 5, respectivamente. Os dados experimentais modelados são apresentados na Tabela 1. Além disso, foi realizada a interpretação dos dados de equilíbrio através da classificação de Giles et al. (1960). 


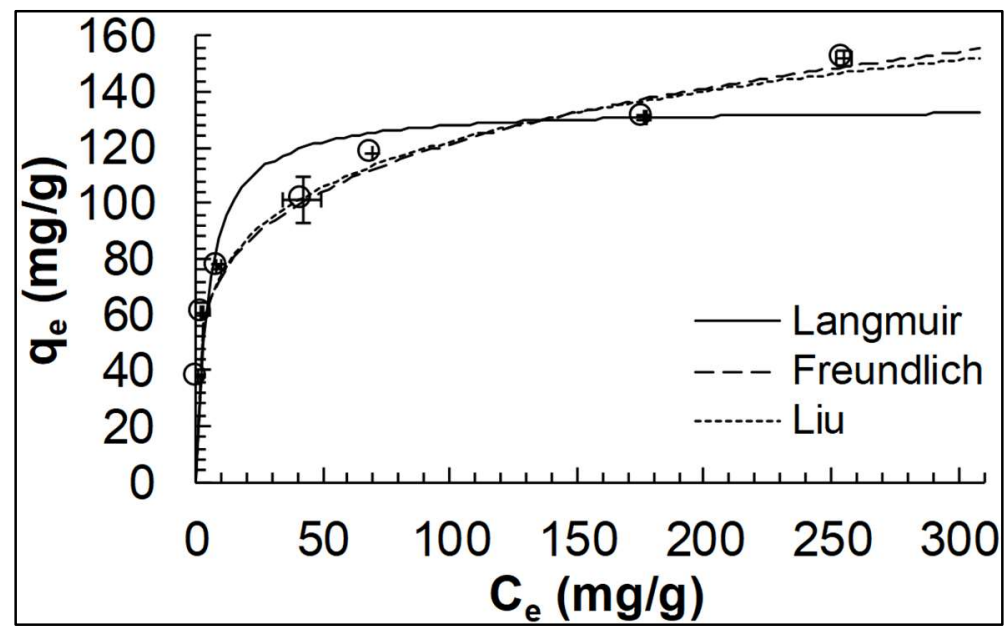

Figura 4: Isoterma de adsorção do corante ABRR pelo xerogel desenvolvido $\left(25^{\circ} \mathrm{C}, 100 \mathrm{rpm}\right.$ e $\left.\mathrm{pH} 3\right)$.

$$
\begin{gathered}
q_{e}=\frac{K_{L} \times q_{m} \times C_{e}}{1+K_{L} \times C_{e}} \\
q_{e}=K_{F} \times C_{e}^{\frac{1}{n F}} \\
q_{e}=\frac{q_{m} \times\left(K_{G} \times C_{e}\right)^{n L}}{1+\left(K_{G} \times C_{e}\right)^{n L}}
\end{gathered}
$$

Sendo, $\mathrm{q}_{\mathrm{m}}$ a capacidade máxima de adsorção $(\mathrm{mg} / \mathrm{g}), \mathrm{K}_{\mathrm{L}}$ a constante de Langmuir $(\mathrm{L} / \mathrm{mg})$, $\mathrm{K}_{\mathrm{F}}$ a constante de Freundlich (mg/g), $\mathrm{nF}$ o fator de heterogeneidade (adimensional), $\mathrm{K}_{\mathrm{G}}$ a constante de Liu (L/mg) e $\mathrm{nL}$ o expoente de Liu (adimensional).

Tabela 1: Parâmetros dos modelos de isotermas obtidos através do ajuste aos dados de equilíbrio de adsorção do corante ABRR pelo xerogel desenvolvido $\left(25^{\circ} \mathrm{C}, 100 \mathrm{rpm}\right.$ e $\left.\mathrm{pH} 3\right)$.

\begin{tabular}{ll|ll|ll}
\hline Langmuir & & Freundlich & & Liu & \\
\hline $\mathrm{K}_{\mathrm{L}}(\mathrm{L} / \mathrm{mg})$ & 0,198 & $\mathrm{~K}_{\mathrm{F}}\left((\mathrm{mg} / \mathrm{g})(\mathrm{L} / \mathrm{mg})^{1 / \mathrm{n}}\right)$ & 44,191 & $\mathrm{~K}_{\mathrm{G}}(\mathrm{L} / \mathrm{mg})$ & 0,0002 \\
$\mathrm{q}_{\mathrm{m}}(\mathrm{mg} / \mathrm{g})$ & 134,383 & $\mathrm{nF}($ adimensional $)$ & 4,564 & $\mathrm{q}_{\mathrm{m}}(\mathrm{mg} / \mathrm{g})$ & 493,609 \\
$\mathrm{R}^{2}$ & 0,863 & $\mathrm{R}^{2}$ & 0,971 & $\mathrm{~nL}($ adimensional) & 0,270 \\
$\mathrm{R}^{2}$ ajustado & 0,851 & $\mathrm{R}^{2}$ ajustado & 0,969 & $\mathrm{R}^{2}$ & 0,973 \\
& & & & $\mathrm{R}^{2}$ ajustado & 0,968 \\
\hline
\end{tabular}

De acordo com a Figura 4 e a classificação de Giles et al. (1960), a isoterma do xerogel classifica-se como tipo L, onde a forma inicial da curva de equilíbrio segue a premissa de que quanto maior a concentração de adsorvato, maior a capacidade de adsorção até que o número de sítios vazios de adsorção seja limitado, havendo concorrência entre as moléculas pelos locais disponíveis. A isoterma do tipo L também indica que a adsorção ocorre devido às forças relativamente fracas, como as forças de Van der Waals (PICCIN et al., 2017). Quanto à subclasse, o formato da isoterma sugere a subclasse 1 (GILES et al., 1960) para representa melhor os dados, indicando que não há formação de monocamada e por não apresentar um platô definido.

Através da Tabela 1 percebe-se que, através do $R^{2}$ o ajuste é melhor para o modelo de Liu $\left(R^{2}\right.$ igual a 0,973), com isso, explicando melhor o comportamento físico apresentado pelos dados experimentais. 0 modelo de Freundlich também apresentou um ajuste satisfatório aos dados experimentais ( $R^{2}$ igual a 0,971 ), justificando a sobreposição desses modelos na Figura 4. Além disso, os valores semelhantes dos coeficientes de determinação ajustado ( $R_{\text {ajustado }}$ ) sugerem que, pela maior simplicidade matemática, o modelo de Freundlich por ser utilizado para correlacionar os dados de equilíbrio. Já o modelo de Langmuir apresentou o menor ajuste aos dados $\left(R^{2}\right.$ igual a 0,863$)$. 
O modelo de equilíbrio de Liu é uma união dos modelos de Langmuir e Freundlich, considerando que a saturação da superfície do adsorvente ocorreria em concentrações mais altas do adsorvato, sendo descrito através de uma equação com três parâmetros (SOPHIA et al., 2019). Esse modelo desconsidera a formação de camada única do modelo de Langmuir e a adsorção infinita do adsorvato do modelo de Freundlich.

A capacidade de adsorção máxima obtida pelo modelo de Liu foi igual a 493,609 mg/g. Esse é um parâmetro teórico, que é obtido apenas em concentrações de adsorvato na fase líquida acima das avaliadas neste estudo, sendo condizente com isotermas do tipo L1, conforme a classificação de Giles et al. (1960). Esta capacidade de adsorção se deve principalmente à interação entre os grupos amina da quitosana e os grupos sulfonados do corante (BLACHNIO et al., 2018). Em condições ácidas os grupos amina da quitosana são protonados (PICCIN et al., 2009). Então, há uma atração com as moléculas do corante aniônico ABRR, principalmente por interações eletrostáticas (PICCIN et al., 2011), como representado na Figura 3.

A Figura 5 apresenta os dados cinéticos de adsorção do corante ABRR pelo xerogel. É possível observar uma rápida adsorção do corante no período de 30 a 45 min, após esse período ocorre uma diminuição na taxa de adsorção, mas não foi verificado uma saturação da monocamada no tempo de até 360 min. Isso indica que ocorreu uma rápida interação entre o corante e os sítios ativos mais externos do xerogel, e ao longo do tempo essa interação pode ocorrer com os grupamentos mais internos da partícula.

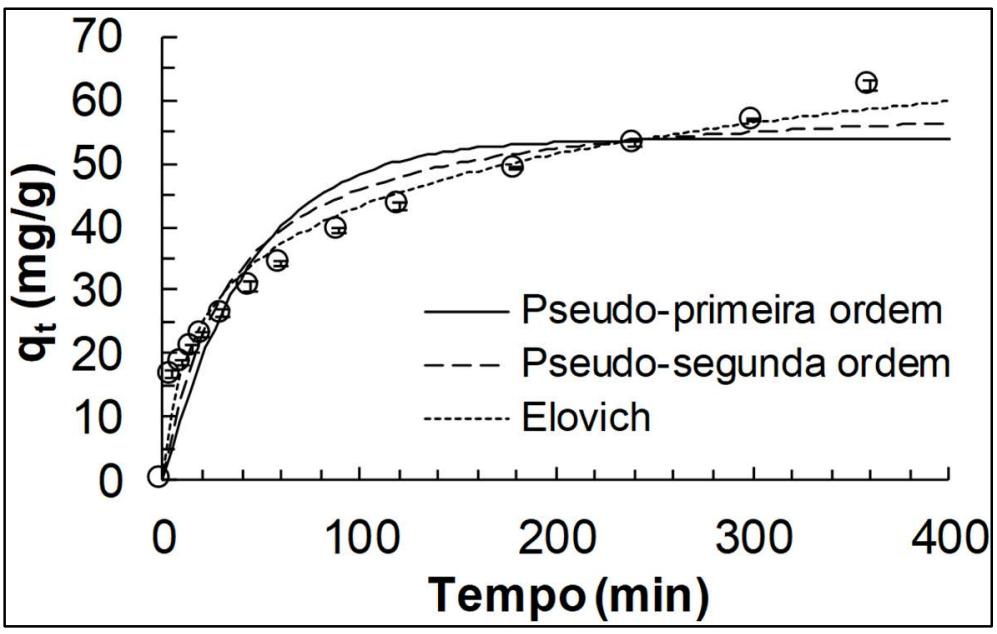

Figura 5: Cinéticas de adsorção do corante ABRR pelo xerogel desenvolvido $\left(25^{\circ} \mathrm{C}, 100 \mathrm{rpm}\right.$ e $\left.\mathrm{pH} 3\right)$.

Para observar os mecanismos de interações predominantes entre o adsorvato e o adsorvente ao longo do tempo e a sua taxa de adsorção, foi verificado o ajuste dos modelos cinéticos pseudo-primeira ordem (LAGERGREN, 1898), pseudo-segunda ordem (HO et al., 2000; HO et al.,1999) e Elovich (ELOVICH et al., 1962) aos dados experimentais, conforme as Equações 6, 7 e 8, respectivamente. Os dados experimentais modelados são apresentados na Tabela 2.

$$
\begin{gathered}
q_{t}=q_{1} \times\left(1-\exp \left(-k_{1} \times t\right)\right) \\
q_{t}=\frac{t}{\left(\frac{1}{k_{2} \times q_{2}^{2}}\right)+\left(\frac{t}{q_{2}}\right)} \\
q_{t}=\frac{1}{a} \times \ln (1+a b t)
\end{gathered}
$$


Sendo, $q_{1}$, q e qt a capacidade de adsorção $(\mathrm{mg} / \mathrm{g})$ no tempo $\mathrm{t}(\mathrm{min}), \mathrm{k}_{1}$ a constante de velocidade de pseudo-primeira ordem $\left(\mathrm{min}^{-1}\right), \mathrm{k}_{2}$ a constante de velocidade de pseudo-segunda ordem (g/mg/min), a sendo a taxa de adsorção inicial ( $\mathrm{mg} / \mathrm{g} / \mathrm{min})$ e b a constante de dessorção $(\mathrm{mg} / \mathrm{g})$.

Tabela 2: Parâmetros dos modelos cinéticos de adsorção do corante ABRR pelo xerogel desenvolvido $\left(25^{\circ} \mathrm{C}, 100 \mathrm{rpm}\right.$ e $\mathrm{pH} 3)$.

\begin{tabular}{ll|ll|ll}
\hline \multicolumn{2}{l|}{ Pseudo-primeira ordem } & \multicolumn{3}{l|}{ Pseudo-segunda ordem } & Elovich \\
\hline $\mathrm{K}_{1}\left(\mathrm{~min}^{-1}\right)$ & 0,023 & $\mathrm{~K}_{2}(\mathrm{~g} / \mathrm{mg} / \mathrm{min})$ & 0,0005 & $\mathrm{a}(\mathrm{g} / \mathrm{mg})$ & 0,082 \\
$\mathrm{q}_{1}(\mathrm{mg} / \mathrm{g})$ & 53,859 & $\mathrm{q}_{2}(\mathrm{mg} / \mathrm{g})$ & 60,878 & $\mathrm{~b}(\mathrm{mg} / \mathrm{g} / \mathrm{min})$ & 4,107 \\
$\mathrm{R}^{2}$ & 0,866 & $\mathrm{R}^{2}$ & 0,927 & $\mathrm{R}^{2}$ & 0,976 \\
$\mathrm{R}^{2}$ ajustado & 0,861 & $\mathrm{R}^{2}$ ajustado & 0,924 & $\mathrm{R}$ & 0,975 \\
\hline
\end{tabular}

Através da Tabela 2, é observado um melhor ajuste do modelo de Elovich aos dados experimentais ( $R^{2}$ igual a 0,976). Os modelos de pseudo-primeira ordem ( $R^{2}$ igual a 0,866$)$ e pseudo-segunda ordem $\left(R^{2}\right.$ igual $0,927)$ apresentaram um menor ajuste aos dados.

O modelo cinético de Elovich se baseia no pressuposto de que o adsorvato é adsorvido na superfície sólida, desprezando os mecanismos de dessorção, e a taxa de adsorção reduz ao longo do tempo devido a uma maior cobertura do adsorvato na superfície (TENG et al., 1999). A equação de Elovich assume que os sítios ativos do adsorvente são heterogêneos e, assim, contêm energias diferentes. Com isso, as atrações eletrostáticas entre a quitosana carregada positivamente em pH ácido $\left(\mathrm{NH}_{3}{ }^{+}\right)$e as moléculas do corante $\mathrm{ABRR}$ carregadas negativamente $\left(\mathrm{SO}_{3}{ }^{-}\right)$, conforme a Figura 3, novamente se evidenciam.

A partir dos dados cinéticos também é possível verificar os mecanismos de transferência de massa na adsorção do corante têxtil, através do modelo de difusão intrapartícula proposto por Weber e Morris (WEBER et al., 1963). $O$ gráfico de Weber e Morris é obtido plotando-se $q_{t}$ versus $t^{0,5}$, conforme a Equação 9 , onde são obtidas porções que representam os diferentes mecanismos de transferência de massa (DOTTO et al., 2012), apresentado na Figura 6.

$$
q_{t}=\operatorname{Kid} \sqrt{t}+I
$$

Sendo, $\mathrm{Kid}\left(\mathrm{mg} / \mathrm{g} / \mathrm{min}^{1 / 2}\right)$ a constante de difusão intrapartícula e I a interceptação.

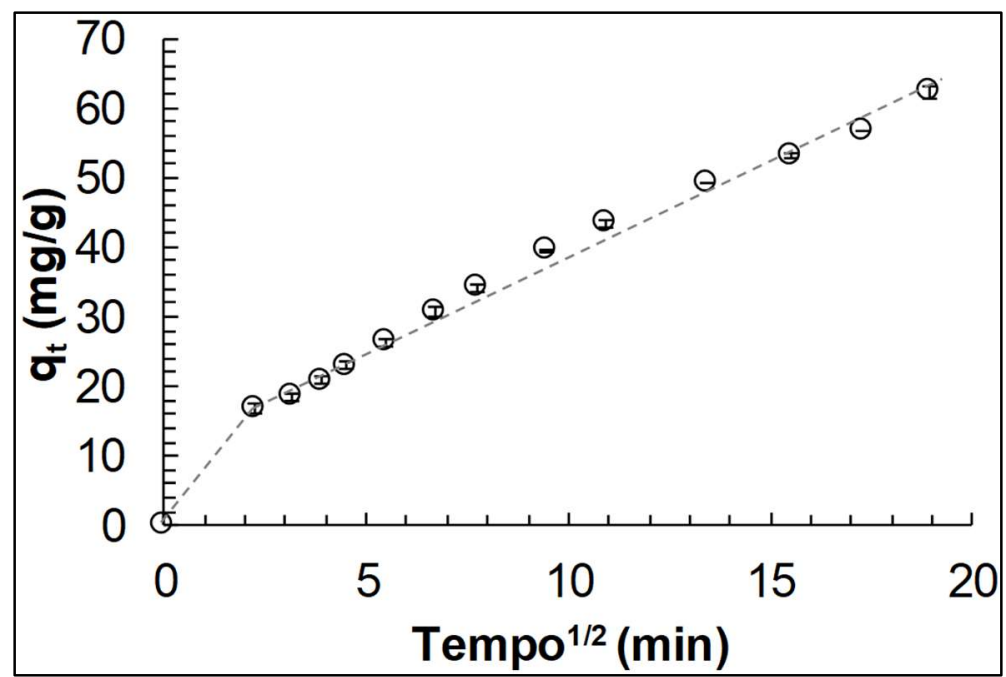

Figura 6: Gráfico de Weber e Morris da adsorção do corante ABRR pelo xerogel desenvolvido $\left(25^{\circ} \mathrm{C}, 100 \mathrm{rpm}\right.$ e pH 3$)$.

No tempo de estudo, há formação de duas porções lineares e cada porção representa um mecanismo distinto da transferência de massa. A primeira porção linear do gráfico indica que houve uma transferência 
de massa externa instantânea (difusão da camada limite) para até 5 min, devido aos sítios ativos da quitosana estarem presentes e livres no xerogel (DOTTO et al., 2012). Após esse tempo, de 5 a 360 min, a segunda porção descreve a etapa de adsorção gradual do corante, sendo possível inferir que, pela linearidade da porção a difusão intrapartícula está envolvida no efeito da taxa de adsorção (JIANG et al., 2018; CRINI et al., 2008). Com isso, a difusão da camada limite e a difusão intrapartícula contribuíram simultaneamente durante o processo de adsorção do corante ABRR.

A formação de compósitos a partir de sílica, produz materiais microestruturados (KOLODYNSKA et al., 2017) que foram preenchidos com a quitosana, podendo ter diminuído a difusividade e favorecendo a linearidade no gráfico de Weber e Morris após 5 min. Porém, a rede de sílica funciona como uma base que afasta os grupos químicos da quitosana, diminuindo assim o impedimento estérico e resultando em um material com alta capacidade de adsorção, fato comprovado pela isoterma.

Segundo Jiang et al. (2018) a modificação da quitosana resulta em materiais adsorventes com uma melhor organização estrutural que favorece a adsorção devido a exposição dos sítios ativos, nesse caso os grupamentos aminas. Nesse estudo, isso pode ter ocorrido através das moléculas do percursor, que se ligaram aos monômeros da quitosana, aumentando sua área superficial e facilitando o acesso aos grupamentos amina (ESSEL et al., 2018). Portanto, a união de um adsorvente com outros materiais através dos métodos de modificação como reticulação, enxerto e a formação de compósitos, resulta em materiais com melhores propriedades de adsorção do que os efeitos dos componentes individuais (AUTA et al., 2014).

\section{CONCLUSÕES}

O compósito proveniente de quitosana e sílica, sintetizado pelo processo sol-gel, demonstrou que a modificação empregada foi efetiva na superação das limitações da quitosana. Isso foi verificado através da alta da capacidade de adsorção em meio ácido, sendo igual a 493,609 mg/g em pH 3 predita pelo modelo de Liu, devido à protonação da quitosana, para a remoção do corante Azul Brilhante de Remazol R. O fenômeno de adsorção ocorreu por difusão da camada limite e intrapartícula simultaneamente.

AGRADECIMENTOS: Ao Conselho Nacional de Desenvolvimento Científico e Tecnológico (CNPQ - Proc. 405311/2016-8), a Coordenação de Aperfeiçoamento de Pessoal de Nível Superior (CAPES - Código financeiro 001) e a Universidade de Passo Fundo (UPF) pelo apoio financeiro.

\section{REFERÊNCIAS}

ABDEL-GAWAD, K. M.; HIFNEY, A. F.; FAWZY, M. A.; GOMAA, M.. Technology optimization of chitosan production from Aspergillus niger biomass and its functional activities. Food Hydrocolloids, v.63, p.593-601, 2017. DOI: http://doi.org/10.1016/i.foodhyd.2016.10.001

AUTA, M.; HAMEEDAB, B. H.. Chitosan-clay composite as highly effective and low-cost adsorbent for batch and fixedbed adsorption of methylene blue. Chemical Engineering Journal, v.237, p.352-361, 2014. DOI: http://doi.org/10.1016/i.cej.2013.09.066

BLACHNIO, M.; BUDNYAK, T. M.; DERYLO-MARCZEWSKA, A.; MARCZEWSKI, A. W.; TERTYKH, V. A.. Chitosan-Silica Hybrid Composites for Removal of Sulfonated Azo Dyes from Aqueous Solutions. Langmuir, v.34, n.6, p.2258-2273, 2018. DOI: http://doi.org/10.1021/acs.langmuir.7b04076

BLACKBURN, R. S.. Natural polysaccharides and their interactions with dye molecules: applications in effluent 
treatment. Environmental Science \& Technology, v.38, p.4905-4909, 2004. DOI: http://doi.org/10.1021/es049972n

BUCKLEY, A. M.; GREENBLATT, M.. The sol-gel preparation of silica gels. Journal of Chemical Education, v.71, n.7, p.599, 1994. DOI: http://doi.org/10.1021/ed071p599

BUDNYAK, T. M.; PYLYPCHUK, I. V.; TERTYKH, V. A.; YANOVSKA, E. S.; KOLODYNSKA, D.. Synthesis and adsorption properties of chitosan-silica nanocomposite prepared by solgel method. Nanoscale Research Letters, v.10, n.1, p.87, 2015. DOI: http://doi.org/10.1186/s11671-014-0722-1

BUDNYAK, T. M.; YANOVSKA, E. S.; KOLODYNSKA, D.; STERNIK, D.; PYLYPCHUK, I. V.; ISCHENKO, M. V.; TERTYKH, V. A.. Preparation and properties of organomineral adsorbent obtained by sol-gel technology. Journal of Thermal Analysis and Calorimetry, v.125, n.3, p.1335-1351, 2016. DOI: http://doi.org/10.1007/s10973-016-5581-9

BURAKOV, A. E.; GALUNIN, E. V.; BORAKOVA, I. V.; KUCHEROVA, A. E.; AGARWAL, S.; TKACHEV, A. G.; GUPTA, V. K.. Adsorption of heavy metals on conventional and nanostructured materials for wastewater treatment purposes: A review. Ecotoxicology and Environmental Safety, v.148, p.702-712, 2018. DOI: http://doi.org/10.1016/j.ecoenv.2017.11.034

CARDOSO, N. F.; LIMA, E. C.; PINTO, I. S.; AMAVISCA, C. V.; ROYER, B.; PINTO, R. B.; ALENCAR, W. S.; PEREIRA, S. F. P.. Application of cupuassu shell as biosorbent for the removal of textile dye from aqueous solution. Journal of Environmental Management, v.92, p.1237-1247, 2011. DOI: http://doi.org/10.1016/j.jenvman.2010.12.010

COPELLO, G. J.; VILANUEVA, M. E.; GONZALEZ, J. A.; EGÜES, S. L.; DIAZ, L. E.. TEOS as an improved alternative for chitosan beads cross-linking: a comparative adsorption Study. Journal of Applied Polymer Science, v.131, n.21, p.41005, 2014. DOI: http://doi.org/10.1002/app.41005

CRINI, G.; BADOT, P. M.. Application of chitosan, a natural aminopolysaccharide, for dye removal from aqueous solutions by adsorption processes using batch studies: a review of recent literature. Progress in Polymer Science, v.33, p.399-447, 2008. DOI:

http://doi.org/10.1016/j.progpolymsci.2007.11.001

DOTTO, G. L.; VIEIRA, M. L. G.; PINTO, L. A. A.. Kinetics and mechanism of tartrazine adsorption onto chitin and chitosan. Industrial \& Engineering Chemistry Research, v.51, n.19, p.6862-6868, 2012. DOI: http://doi.org/10.1021/ie2030757

ELOVICH, S. Y.; LARINOV, O. G.. Theory of adsorption from solutions of nonelectrolytes on solid (I) equation adsorption from solutions and the analysis of its simplest form, (II) verification of the equation of adsorption isotherm from solutions. Izvestiya Akademii Nauk SSSR, Otdelenie Khimicheskikh Nauk, v.2, p.209-216, 1962.

ELTAWEIL, A. S.; ELGARHY, G. S.; EL-SUBRUITI, G. M.; OMER, A. M.. Carboxymethyl cellulose/carboxylated graphene oxide composite microbeads for efficient adsorption of cationic methylene blue dye. International Journal of Biological Macromolecules, v.154, p.307-318, 2020. DOI:

\section{http://doi.org/10.1016/i.ijbiomac.2020.03.122}

ESSEL, T. Y. A.; KOOMSON, A.; SENIAGYA, M. P. O.; COBBOLD, G. P.; KWOFIE, S. K.; ASIMENG, B. O.; ARTHUR, P. K.; AWANDARE, G.; TIBURU, E. K.. Chitosan Composites Synthesized Using Acetic Acid and Tetraethylorthosilicate Respond Differently to Methylene Blue Adsorption. Polymers, v.10, p.466, 2018. DOI: http://doi.org/10.3390/polym10050466

FRANCO, D. S. P.; PICCIN, J. S.; LIMA, E. C; DOTTO, G. L.. Interpretations about methylene blue adsorption by surface modified chitin using the statistical physics treatment. Adsorption, v.21, p.557-564, 2015. DOI: http://doi.org/10.1007/s10450-015-9699-z

FIOL, N.; VILLAESCUSA, I.. Determination of sorbent point zero charge: usefulness in sorption studies. Environmental Chemistry Letters, v.7, n.1, p.79-84, 2009. DOI: http://doi.org/10.1007/s10311-008-0139-0

FREUNDLICH, H. M. F.. Over the adsorption in solution. Journal of Physical Chemistry, v.57, n.385471, p.1100-1107, 1906.

FUTALAN, C. M.; KAN, C. C.; DALIDA, M. L.; PASCUA, C.; WAN, M. W.. Fixed-bed column studies on the removal of copper using chitosan immobilized on bentonite. Carbohydrate Polymers, v.83, p.697-704, 2011. DOI: http://doi.org/10.1016/j.carbpol.2010.08.043

GILES, C. H.; MACEWAN, T. H.; NAKHWA, S. N.; SMITH, D. Studies in adsorption. Part XI. A system of classification of solution adsorption isotherms, and its use in diagnosis of adsorption mechanisms and in measurement of specific surface areas of solids. Journal of the Chemical Society, p.3973-3993, 1960. DOI:

http://doi.org/10.1039/JR9600003973

GISI, S.; LOFRANO, G.; GRASSI, M.; NOTARNICOLA, M.. Characteristics and adsorption capacities of low-cost sorbents for wastewater treatment: A review. Sustainable Materials and Technologies, v.9, p.10-40, 2016. DOI: http://doi.org/10.1016/i.susmat.2016.06.002

GOMAA, M.; HIFNEY, A.; FAWZY, M. A.; ABDEL-GAWAD, K. $M$.. Use of seaweed and filamentous fungus derived polysaccharides in the development of alginate-chitosan edible films containing fucoidan: Study of moisture sorption, polyphenol release and antioxidant properties. Food Hydrocolloids, v.82, p.239-247, 2018. DOI: http://doi.org/10.1016/j.foodhyd.2018.03.056

GOMES, C. S.; PICCIN, J. S.; GUTTERRES, M.. Optimizing adsorption parameters in tannery-dye-containing effluent treatment with leather shaving waste. Process Safety and Environmental Protection, v.99, p.98-106, 2016. DOI: http://doi.org/10.1016/i.psep.2015.10.013

HENCH, L. L.; WEST, J. K.. The Sol-Gel process. Chemical Reviews, v.90, p.33-72, 1990. DOI: http://doi.org/10.1021/cr00099a003

HO, Y. S.; MCKAY, G.. Pseudo-second order model for sorption processes. Process Biochemistry, v.34, p.451-465, 1999. DOI: http://doi.org/10.1016/S0032-9592(98)00112-5 
HO, Y. S.; MCKAY, G.. The kinetics of sorption of divalent metal ions onto sphagnum moss peat. Water Research, v.34, n.3, p.735-742, 2000. DOI: http://doi.org/10.1016/s0043$\underline{1354(99) 00232-8}$

IBANESCU, A.; ALEXANDRICA, M. C.; HRITCU, D.; CHISCAN, O.; POPA, M. I.. Magnetite/chitosan composite particles as adsorbents for Reactive Blue 19 dye. Green Materials, v.6, n.4, p.149-156, 2018. DOI:

http://doi.org/10.1680/igrma.18.00039

JIANG, Y.; LIU, B.; XU, J.; PAN, K.; HOU, H.; HU, J.; YANG, J.. Cross-linked chitosan/ $\beta$-cyclodextrin composite for selective removal of methyl orange: Adsorption performance and mechanism. Carbohydrate Polymers, v.182, p.106-114, 2018. DOI: http://doi.org/10.1016/j.carbpol.2017.10.097

KLEIN, L. C.. Sol-gel processing of silicates. Annual Review of Materials Science, v.15, n.1, p.227-248, 1985. DOI: http://doi.org/10.1146/annurev.ms.15.080185.001303

KOLODYNSKA, D.. Chitosan as an effective low-cost sorbent of heavy metal complexes with the polyaspartic acid. Chemical Engineering Journal, v.173, p.520-529, 2011. DOI: http://doi.org/10.1016/i.cej.2011.08.025

KOLODYNSKA, D.; BUDNYAK, T. M; HUBICKI, Z.; TERTYKH, V. A.. Sol-Gel Derived Organic-Inorganic Hybrid Ceramic Materials for Heavy Metal Removal. In: MISHRA, AK.. Sol-gel Based Nanoceramic Materials: Preparation, Properties and Applications. Cham: Springer, 2017. p.253-274. DOI: http://doi.org/10.1007/978-3-319-49512-5 9

KYZAS, G. Z.; KOSTOGLOU, M.; LAZARIDIS, N. K.; LAMBROPOULOU, D. A.; BIKIARIS, D. N.. Environmental friendly technology for the removal of pharmaceutical contaminants from wastewaters using modified chitosan adsorbents. Chemical Engineering Journal, v.222, p.248-258, 2013. DOI: http://doi.org/10.1016/j.cej.2013.02.048

LAGERGREN, S.. Zur theorie der sogenannten adsorption geloster stoffe, kungliga svenska vetenskapsakademiens. Handlingar, v.24, n.4, p.1-39, 1898.

LANGMUIR, I.. The adsorption of gases on plane surfaces of glass, mica and platinum. Journal of the American Chemical society, v.40, n.9, p.1361-1403, 1918.

LESSA, E. F.; NUNES, M. L.; FAJARDO, A. R.. Chitosan/waste coffee-grounds composite: An efficient and eco-friendly adsorbent for removal of pharmaceutical contaminants from water. Carbohydrate Polymers, v.189, p.257-266, 2018. DOI: http://doi.org/10.1016/j.carbpol.2018.02.018

LIU, Y.; XU, H.; YANG, S. F.; TAY, J. H.. A general model for biosorption of $\mathrm{Cd}^{2+}, \mathrm{Cu}^{2+}$ and $\mathrm{Zn}^{2+}$ by aerobic granules. Journal of Biotechnology, v.102, n.3, p.233-239, 2003. DOI: http://doi.org/10.1016/S0168-1656(03)00030-0

LIU, Y.; LIU, R.; LI, M.; YU, F.; HE, C.. Removal of pharmaceuticals by novel magnetic genipin-crosslinked chitosan/graphene oxide- $\mathrm{SO}_{3} \mathrm{H}$ composite. Carbohydrate Polymers, v.220, p.141-148, 2019. DOI: http://doi.org/10.1016/j.carbpol.2019.05.060
LIZARDI-MENDOZA, J.; MONAL, W. M. A.; VALENCIA, F. M. G.. Chemical characteristics and functional properties of chitosan.In: BAUTISTA-BAÑOS, S.; ROMANAZZI, G.; JIMÉNEZ$A P A R I C I O, A .$. Chitosan in the preservation of agricultural commodities. Academic Press, 2016. p.3-31. DOI: http://doi.org/10.1016/B978-0-12-802735-6.00001-X

MOURA, J. M.; FARIAS, B. S.; RODRIGUES, D. A. S.; MOURA, C. M.; DOTTO, G. L.; PINTO, L. A. A.. Preparation of Chitosan with Different Characteristics and Its Application for Biofilms Production. Journal of Polymers and the Environment, v.23, n.4, p.470-477, 2015. DOI: http://doi.org/10.1007/s10924$\underline{015-0730-y}$

MUÑOZ, G.; VALENCIA, C.; VALDERRUTEN, N.; RUIZDURÁNTEZ, E.; ZULUAGA, F.. Extraction of chitosan from Aspergillus niger mycelium and synthesis of hydrogels for controlled release of betahistine. Reactive and Functional Polymers, v.91-92, p.1-10, 2015. DOI:

http://doi.org/10.1016/j.reactfunctpolym.2015.03.008

NEWCOMBE, G.; HAYES, R.; DRIKAS, M.. Granular activated carbon: importance of surface properties in the adsorption of naturally occurring organics. Colloids and Surfaces A: Physicochemical and Engineering Aspects, v.78, p.65-71, 1993. DOI: http://doi.org/10.1016/0927-7757(93)80311-2

NGAH, W. S. W.; TEONG, L. C.; HANAFIAH, M. A. K. M.. Adsorption of dyes and heavy metal ions by chitosan composites: A review. Carbohydrate Polymers, v.83, p.14461456, 2011. DOI:

http://doi.org/10.1016/i.carbpol.2010.11.004

PERDIGOTO, L. N.; MARTINS, R. C.; ROCHA, N.; QUINA, M. J.; GANDO-FERREIRA, L.; PATRÍCIO, R.; DURÃESA, L.. Application of hydrophobic silica based aerogels and xerogels for removal of toxic organic compounds from aqueous solutions. Journal of Colloid and Interface Science, v.380, n.1, p.134-140, 2012. DOI:

http://doi.org/10.1016/j.jcis.2012.04.062

PICCIN, J. S.; VIEIRA, M. L. G.; GONÇALVES, J. O.; DOTTO, G. L.; PINTO, L. A. A.. Adsorption of FD\&C Red No. 40 by chitosan: Isotherms analysis. Journal of Food Engineering, v.95, p.16-20, 2009. DOI:

http://doi.org/10.1016/j.jfoodeng.2009.03.017

PICCIN, J. S; DOTTO, G. L.; VIEIRA, M. L. G.; PINTO, L. A. A.. Kinetics and mechanism of the food dye FD\&C Red 40 adsorption onto chitosan.Journal of Chemical \& Engineering, v.56, n.10, p.3759-3765, 2011. DOI: http://doi.org/10.1021/je200388s

PICCIN, J. S.; FERIS, L. A.; COOPER, M.; GUTTERRES, M.. Dye adsorption by leather waste: mechanism diffusion, nature studies, and thermodynamic data. Journal of Chemical \& Engineering Data, v.58, n.4, p.873-882, 2013. DOI: http://doi.org/10.1021/ie301076n

PICCIN, J. S.; CADAVAL JUNIOR, T. R. S.; PINTO, L. A. A.; DOTTO, G. L.. Adsorption Isotherms in Liquid Phase: Experimental, Modeling, and Interpretations. In: BONILLAPETRICIOLET, A.; MENDONZA-CASTILLO, D. I.; REYNEL-ÁVILA, H. E.. Adsorption Processes for water treatment and purification. Cham: Springer, 2017. p.19-51. DOI: http://doi.org/10.1007/978-3-319-58136-12 
REPO, E.; WARCHOt, J. K.; BHATNAGAR, A.; SILLANPÄÄ, M.. Heavy metals adsorption by novel EDTA-modified chitosansilica hybrid materials. Journal of Colloid and Interface Science, v.358, p.261-267, 2011. DOI: http://doi.org/10.1016/j.jcis.2011.02.059

ROSSI, A.; RIGON, M. R.; ZAPAROLI, M.; BRAIDO, R. D.; COLLA, L. M.; DOTTO, G. L.; PICCIN, J. S.. Chromium (VI) biosorption by Saccharomyces cerevisiae subjected to chemical and thermal treatments. Environmental Science and Pollution Research, v.25, p.19179-19186, 2018. DOI: http://doi.org/0.1007/s11356-018-2377-4

SOPHIA, A. C.; ARFIN, T.; LIMA, E. C.. Recent Developments in Adsorption of Dyes Using Graphene Based Nanomaterials. In: NAUSHAD, M.. A New Generation Material Graphene: Applications in Water Technology. Cham: Springer, 2019. p.439-471. DOI: http://doi.org/10.1007/978-3-319-75484$\underline{018}$

SUBRAMANI, S. E.; THINKARAN, N.. Isotherm, kinetic and thermodynamic studies on the adsorption behaviour of textile dyes onto chitosan. Process Safety and Environmental Protection, v.160, p.1-10, 2017. DOI: http://doi.org/10.1016/j.psep.2016.11.024
TENG, H.; HSIEH, C. T.. Activation energy for oxygen chemisorption on carbon at low temperatures. Industrial \& Engineering Chemistry Research, v.38, n.1, p.292-297, 1999. DOI: http://doi.org/10.1021/ie980107i

VAKILI, M.; RAFATULLAH, M.; SALAMATINIA, B.; ABDULLAH, A. Z.; IBRAHIM, M. H.; TAN, K. B.; GHOLAMI, Z.; MOUZGAR, P.. Application of chitosan and its derivatives as adsorbents for dye removal from water and wastewater: A review. Carbohydrate Polymers, v.113, p.115-130, 2014. DOI: http://doi.org/10.1016/j.carbpol.2014.07.007

WEBER, W. J.; MORRIS, J. C.. Kinetics of adsorption on carbon from solutions. Journal of the Sanitary Engineering Division, v.89, n.2, p.31-60, 1963.

WESKA, R. F.; MOURA, J. M.; BATISTA, L. M.; RIZZI, J.; PINTO, L. A. A.. Optimization of deacetylation in the production of chitosan from shrimp wastes: Use of response surface methodology. Journal of Food Engineering, v.80, p.749-75, 2007. DOI: http://doi.org/10.1016/j.jfoodeng.2006.02.006

ZOU, H.; WU, S.; SHEN, J.. Polymer/Silica Nanocomposites: Preparation, Characterization, Properties, and Applications. Chemical Reviews, v.108, n.9, p.3893-3957, 2008. DOI: http://doi.org/10.1021/cr068035q

A CBPC - Companhia Brasileira de Produção Científica (CNPJ: 11.221.422/0001-03) detém os direitos materiais desta publicação. Os direitos referem-se à publicação do trabalho em qualquer parte do mundo, incluindo os direitos às renovações, expansões e disseminações da contribuição, bem como outros direitos subsidiários. Todos os trabalhos publicados eletronicamente poderão posteriormente ser publicados em coletâneas impressas sob coordenação da Sustenere Publishing, da Companhia Brasileira de Produção Científica e seus parceiros autorizados. Os (as) autores (as) preservam os direitos autorais, mas não têm permissão para a publicação da contribuição em outro meio, impresso ou digital, em português ou em tradução. 